\title{
Complement Enzyme Antibody Method and Application of Nucleic Acid Research by Enzyme Antibody Method
}

\author{
Ikuo Suzuki \\ Laboratory of Ultrastructure Research Aichi Cancer Center, \\ Research Institute, Nagoya, 464
}

The complement required antigen-antibody reaction system with anti-complement (C3) were successfully applied to the detection of the tumor associated antigen of Burkitt lymphoma (EBNA) which was not able to detect by routine enzyme antibody method. The advantages of the method were not only able to detect the antigen with IgG and IgM class antibodies but also expected the higher sensibility than that of the routine method.

The detection of single stranded DNA in cell nucleus in situ was performed by the enzyme antibody method using the anti-thymine antibody. Anti-thymine was prepared by conjugation of carrier protein (BSA). It was proved that the antibody only reacted with single stranded DNA but not with double stranded DNA and RNA. This fact was also confirmed by the experiments using heteroduplex of DNA. The positive reaction products were detected in the nuclei of regenerating rat liver cell and of culture cell. The characterization of single stranded region was performed using bacteria which were known about DNA replication.

\section{Vaccinia Virus Infection in vitro Studied by Peroxidase-Labeled Antibody Method}

\author{
Hiroyuki Мгуамото
}

Department of Pathology, Research Institute for Microbial Diseases, Osaka University, Osaka 565

Ultrastructural studies on multiplication and development of various viruses in infected cells are numerous. Principal steps so far estabulished with these studies are as follows: 1) attachment of virus to a cell surface, 2) engulfment of the virus by the cell, 3) disappearance of the viral coating (uncoating), 4) assembly of new infectious viruses in inclusion bodies, 5) release of the infectious viruses from the cell. However, the steps between the uncoating(Step 3) and the appearance of new virus(Step 4) are unobservable with routine electron microscopic techniques.

In this study, the sequence of the infection of vaccinia virus and the appearance of new infectious virus were investigated with particular emphasis on the stages between the uncoating and the reappearance in LLC-MK ${ }_{2}$ cells in vitro with the peroxidase-labeled antibody method.

Rabbit antisera against LS antigen were used throughout this study. The rabbit antibody against the LS antigen was digested with pepsin and the Fab' of the 
antibodies were isolated by column chromatography. The Fab' antibodies were then labeled with horseradish peroxidase with the method described by Dr. Kawaoi. LLC-MK $\mathrm{K}_{2}$ cells were exposed to isolated vaccinia viruses and the fate of the LS antigen was followed with the labeled antibody. At various times following the exposure of vaccinia virus, the cells were fixed with periodate-lysine-paraformaldehyde (PLP) fixative as described by Dr. Nakane. The peroxidase-labeled anti-LS were then used to localize the antigen both at light and electron microscopic levels.

At the light microscopic level, the viral antigen was visible as a small rod on the cell surface and in the cytoplasm during the first four hours. The intervals between the four and ten hours following the virus exposure, the antigen was found diffusely throughout the cytoplasm with focal accumulation as the duration of the exposure was increased. This phenomenon may be interpreted as the migration of the antigen from the cytoplasm to the inclusion bodies for the formation of new viruses. Some viral antigen remained on the cell surface throughout the duration of the experiments.

At the electron microscopic level, the viral antigen was found in the outer layer of coat when the viruses were situated on the cell surface, in the process of engulfment by the cell, and immidiately after the penetration into the cytoplasm. Within two hours after the inoculation, the coat began to disappear and the viral antigen was found adjacent to the plasma membrane. Three of four layers of flat saccules or thread like materials were now found in the center of the virus particle without the LS antigen. This was followed with polarization of the antigen at the ends of the thread. As this polarization was progressed, the number of thread was increased to about ten or more. The LS antigen was also found at the ends of these newly formed thread. The polarized LS antigen masses begins to increase as the thread begins to disappear. Four hours after the inoculation, the masses of LS antigen continue to increase and begins to look like primitive inclusion bodies. During this early phases of infection, some viral particles with antigen were found also found within lysosome like membrane bound vacuoles. The size and shapes of the viruses suggest that they are being digested by the lysosomal enzyme. From six to ten hours after the infection, new viruses were began to be formed within the inclusion bodies. First visible sign of formation of new virus was the formation of new coat with LS antigen. Eventurally new generation of viruses in their complete form were found within the inclusion bodies.

In this study, we are able to follow the complete life cycle of vaccinia virus. The appearance of the thread like material within the parent viral particle may corresponds to the DNA like material found within the mature virus which are not visible with routine electron microcspy when viruses were fixed with ordinal fixatives. 